Muñoz-Cutuli, L.; de la Vega-Marcos, R. y Díaz-del-Cueto, M. (2020) Validation of an Instrument on Tactical-Offensive Knowledge for Players with Intellectual Disability. Revista Internacional de Medicina y Ciencias de la Actividad Física y el Deporte vol. 20 (79) pp. 487-506 Http://cdeporte.rediris.es/revista/revista79/artherramienta1181.htm

DOI: http://doi.org/10.15366/rimcafd2020.79.007

\title{
ORIGINAL
}

\section{VALIDACIÓN DE UN INSTRUMENTO SOBRE CONOCIMIENTO TÁCTICO-OFENSIVO PARA JUGADORES CON DISCAPACIDAD INTELECTUAL}

\section{VALIDATION OF AN INSTRUMENT ON TACTICAL- OFFENSIVE KNOWLEDGE FOR PLAYERS WITH INTELLECTUAL DISABILITY}

\author{
Muñoz-Cutuli, L. ${ }^{\text {; }}$ de la Vega-Marcos, R. $^{2}$ y Díaz-del-Cueto, M. ${ }^{2}$ \\ 1 Profesor Titular del Departamento de Primaria. La Salle Centro Universitario Madrid \\ luismc@lasallecampus.es \\ 2 Profesor Doctor del Departamento de Educación Física, Deporte y Motricidad Humana, \\ Facultad de Educación de la Universidad Autónoma de Madrid ricardo.delavega@uam.es, \\ mario.diaz@uam.es
}

Código UNESCO / UNESCO code: 5899 Otras especialidades pedagógicas (Educación Física y Deporte adaptado).

Clasificación Consejo de Europa / Council of Europe classification: 4. Educación Física y deporte comparado / Comparative physical Education and sport

Recibido 24 de septiembre de 2018 Received September 24, 2018 Aceptado 7 de julio de 2019 Accepted July 7, 2019

\section{RESUMEN}

El objetivo de este trabajo se centra en construir y validar una herramienta que permita, de forma fiable, evaluar el conocimiento de base de jugadores con discapacidad intelectual leve en aspectos de táctica ofensiva en FS, permitiendo usarlo como evaluación inicial complementaria que guíe el proceso de entrenamiento. El tamaño muestral fue de 68 jugadores $\left(M_{\text {edad }}=27 ; S D_{\text {edad }}=9.06\right.$

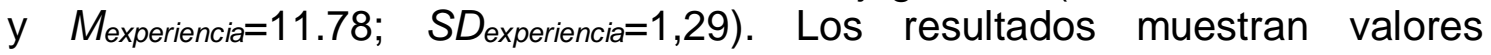
adecuados de consistencia interna y de fiabilidad $(\alpha=0.64, \omega=0.74, I C C=0.64$ y test-retest). De manera complementaria, se realizó un análisis cualitativo, mediante una entrevista colectiva a un grupo de expertos, sobre la utilidad del instrumento en esta población de deportistas. Los resultados permiten establecer una sólida base para el empleo de este instrumento en futuros estudios. 
PALABRAS CLAVE: discapacidad intelectual, toma de decisiones, deporte adaptado, fútbol sala.

\begin{abstract}
The objective of this work is to build and validate a tool that allows, reliably, evaluate the base knowledge of players with mild intellectual impairment in aspects of offensive tactical in FS, so that it can be used as an additional initial evaluation to guide the training process of coaches. The sample size is constituted by 68 subjects $\left(M_{\text {age }}=27 ; S D_{\text {age }}=9.06\right.$ y $M_{\text {experience }}=11.78$;

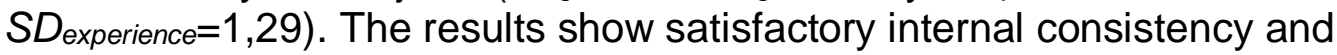
reliability values ( $\alpha=0.64, \omega=0.74, \mathrm{ICC}=0.64$ and test-retest). In a complementary manner, a qualitative analysis was carried out, through a collective interview with a group of experts, about the utility of the instrument in this population of athletes. The results allow to establish a solid base for the use of this instrument in future studies.
\end{abstract}

KEYWORDS: Intellectual disabilities, decision making, adapted sport, futsal.

\title{
INTRODUCCIÓN
}

Los estudios realizados en deporte adaptado dirigido a personas con discapacidad intelectual leve (DIL), defienden que estos deportistas muestran una resolución inferior en diferentes tareas cognitivas pero mejorables tras un entrenamiento específico. Se destaca la importancia de evaluar los componentes cognitivos con el conocimiento básico de la táctica del deportista, relacionado con la línea de investigación anglosajona de MacMahon \& McPherson (2009) sobre el "conocimiento de base" o "knowledge-base", para adecuar la planificación de dichos entrenamientos, y de esta manera optimizar su rendimiento deportivo (Meilán, Salgado, Arana, Carro, \& Jenaro, 2008; Van Biesen, Mactavish, \& Vanlandewijck, 2014).

Como apuntan Wehmeyer \& Obremski (2010), la resolución de tareas requiere una adecuada toma de decisiones que puede verse influenciada por limitaciones en el funcionamiento intelectual. Estas limitaciones incluyen dificultades en la comprensión de ideas, el razonamiento, la resolución de problemas, la planificación, el pensamiento abstracto y el aprovechamiento de la experiencia para la adquisición de nuevos aprendizajes. Todas ellas, son aspectos relevantes para el desempeño óptimo de aprendizajes de naturaleza táctica y decisional (De la Vega, 2003).

En este sentido, la toma de decisiones puede conceptualizarse como un proceso complejo desde la perspectiva psicológica y motriz (De la Vega, Del Valle, Maldonado, \& Moreno, 2008), en el que se expone al sujeto a la elección de varias alternativas condicionadas por el contexto de juego, debiendo elegir una de ellas para conseguir un resultado, el cual puede ser adecuado o erróneo (Elwyn \& Miron-Shatz, 2010; Lim \& Jain, 2010; McPherson \& Kernodle, 2007; Ruiz-Pérez \& Arruza, 2005). 
Ubicados desde la perspectiva de la psicología cognitiva, Anderson (1987) ya mencionaba como la estructura de conocimiento, en nuestro caso del jugador de fútbol sala (FS), se forma en base a dos tipos: el conocimiento declarativo y el conocimiento procedimental. Esta división se basa en la diferenciación entre el saber cómo hacer (procedimental), es decir, la ejecución del movimiento motor adecuado para resolver el problema, y el saber qué hacer (declarativo), es decir, la capacidad de determinar la posibilidad de elección en un cuerpo organizado de la información (Abernethy, Thomas, \& Thomas, 1993; Fernández, Moreno, Gil, Claver, \& Moreno, 2014).

El desarrollo del conocimiento declarativo, teniendo en cuenta a autores como Gréhaigne, Wallian \& Godbout (2005), López-Ros (2011), Raab (2003) y RuizPérez \& Arruza (2005), se asocia estrechamente al dominio del pensamiento táctico, considerándose la capacidad que tiene el jugador de conocer y manifestar la mejor decisión sobre la acción motora a llevar a cabo en cada situación de juego. El pensamiento táctico permitiría desarrollar el conocimiento procedimental (Allard, Deakin, Parker, \& Rodgers, 1993; De la Vega et al., 2008; Matias \& Greco, 2010).

Tras una detallada búsqueda bibliográfica mediante el empleo de las bases de datos Web of Science, SportDiscus, SciELO, Redalyc, Scopus, ERIC, Dialnet y Pubmed, con la utilización de las palabras clave como intellectual disabilities, decision making, adapted sport y futsal; resulta evidente la reducida importancia que se le ha otorgado al estudio de los elementos decisionales en el deporte con poblaciones con DIL. En este sentido, tomando como referente el deporte del baloncesto, Pérez-Tejero, Polo-Más, Pinilla, \& Coterón-López (2017) establecen que los entrenadores y árbitros de su estudio consideran la táctica individual ofensiva como el elemento que presenta mayores limitaciones en los jugadores con discapacidad intelectual (DI). Estas limitaciones, según Pinilla, Pérez-Tejero, Van Biesen, \& Vanlandewijck (2016), pueden explicarse por las dificultades que tienen los jugadores con DI al llevar a cabo tácticas individuales y colectivas en las que se requieren habilidades cognitivas para percibir, decidir y ejecutar correctamente.

En el deporte del FS, como contexto de estudio, el antecedente más relevante que se ha encontrado es la investigación realizada por Moya, Villagra \& García (2006). En este trabajo, bajo el paradigma ecológico, se encontró relación entre los niveles técnicos y tácticos de los jugadores con DI y el rendimiento obtenido, concluyendo en la importancia de individualizar el análisis y evaluación de las capacidades y competencias de los jugadores para optimizar el proceso de entrenamiento deportivo.

Como se puede apreciar en la tabla 1, se muestran otros estudios realizados hasta la fecha sobre la toma de decisiones en el deporte del baloncesto y del tenis de mesa pero centrados en el análisis del plano procedimental de la acción, promovido también por la gran influencia actual del paradigma ecológico (Araújo \& Davids, 2011; Kelso, 2012), basando los estudios en las formas de adaptación de los jugadores en función de las situaciones de juego evaluadas (Davids, Renshaw, Pinder, Araújo, \& Vilar, 2012). 
Tabla 1: Investigaciones realizadas sobre capacidad táctica en deportistas con DI.

\begin{tabular}{|c|c|}
\hline \multicolumn{2}{|r|}{ BALONCESTO } \\
\hline ESTUDIO & CONCLUSIÓN \\
\hline $\begin{array}{l}\text { Guidetti, Franciosi, } \\
\text { Emerenziani, Gallotta, \& } \\
\text { Baldari (2009) }\end{array}$ & $\begin{array}{l}\text { Este estudio demostró que un programa de entrenamiento de } \\
\text { baloncesto específico consiguió una mejora general en el } \\
\text { rendimiento deportivo y en las habilidades de baloncesto para } \\
\text { todos los atletas con DI. A su vez, la batería de prueba propuesta } \\
\text { en esta investigación podría ser útil para evaluar si el nivel de } \\
\text { habilidad técnica y táctica en base a cuatro niveles de dificultad } \\
\text { para el manejo de balón, recepción, pases y tiros; individual y de } \\
\text { equipo sería adecuada para participar en una categoría de } \\
\text { campeonato específica. }\end{array}$ \\
\hline $\begin{array}{l}\text { Franciosi, Gallotta, } \\
\text { Baldari, \& Emerenziani } \\
\text { (2012) }\end{array}$ & $\begin{array}{l}\text { Confirmaron los resultados de estudios anteriores, mostrando } \\
\text { mejoras significativas en los jugadores de baloncesto con DI tras } \\
\text { un programa de entrenamiento en las habilidades de manejo de } \\
\text { balón, recepción, pases y tiros. Además, se encontró que la } \\
\text { batería de prueba de baloncesto podría ser útil para mejorar y } \\
\text { monitorear el entrenamiento en los jugadores. }\end{array}$ \\
\hline $\begin{array}{l}\text { Pérez-Tejero, Pinilla \& } \\
\text { Vanlandewijck (2015) }\end{array}$ & $\begin{array}{l}\text { Faltan de estudios sobre aspectos relacionados con la toma de } \\
\text { decisiones en el juego y su comparativa con jugadores sin DI. }\end{array}$ \\
\hline Pinilla et al. (2016) & $\begin{array}{l}\text { Las limitaciones en la capacidad de resolver problemas por parte } \\
\text { de las personas con DI, influyendo negativamente en las } \\
\text { decisiones tomadas frente a situaciones tácticas de juego, } \\
\text { pudiendo diferenciar entre jugadores con o sin DI. }\end{array}$ \\
\hline $\begin{array}{l}\text { Pinilla, Pérez-Tejero \& } \\
\text { Van Biesen (2017) }\end{array}$ & $\begin{array}{l}\text { Los jugadores con DI alcanzan peor nivel de su rendimiento frente } \\
\text { a los jugadores sin DI. Las limitaciones cognitivas que presentan } \\
\text { posiblemente influyan de forma negativa en la interpretación de } \\
\text { las acciones de juego, en la toma de decisiones y en la } \\
\text { elaboración de acciones en equipo. }\end{array}$ \\
\hline \multicolumn{2}{|r|}{ TENIS DE MESA } \\
\hline ESTUDIO & CONCLUSIÓN \\
\hline Van Biesen et al., (2014) & $\begin{array}{l}\text { Los jugadores con DI tienen una capacidad táctica más reducida } \\
\text { en la devolución del saque para anotar punto con respecto a } \\
\text { jugadores sin DI. }\end{array}$ \\
\hline $\begin{array}{l}\text { Van Biesen, Mactavish, } \\
\text { Kerremans \& } \\
\text { Vanlandewijck (2016) }\end{array}$ & $\begin{array}{l}\text { Las limitaciones que presentan los jugadores con DI en los } \\
\text { aspectos cognitivos les impiden competir en igualdad de } \\
\text { condiciones frente a los jugadores sin DI. }\end{array}$ \\
\hline
\end{tabular}

Este enfoque, si bien es relevante y muy pertinente, no agota la importancia de contar con instrumentos que, en el contexto de la DIL, permitan interpretar el nivel de conocimiento de base que tienen los jugadores para poder vertebrar los contenidos de entrenamiento posteriores que se deben establecer en función de los primeros. Es importante, desde este enfoque, interpretar la relevancia dialéctica entre el plano de la acción (procedimental), y el plano de la representación (conceptual), puesto que la mejor adaptación del jugador vendrá cuando ambos se equilibran, superando en el plano representacional limitaciones procedimentales de orden técnico y táctico (De la Vega, 2003; De la Vega et al., 2008).

Es precisamente en este sentido en el que esta investigación cobra un sentido esencial, puesto que del conocimiento de la relevancia del plano declarativo/conceptual, es desde donde pensamos que surge la adaptación del 
organismo al medio en una primera instancia, buscando emplear todos sus recursos disponibles, obviamente también motrices, para generar nuevos niveles de conocimiento tanto declarativo como procedimental. En este mismo sentido, estudios previos desde un enfoque neurocognitivo (Calvo-Merino, Glaser, Grèzes, Passingham, \& Haggard, 2004), han ofrecido respaldo a la idea de que cuando se observa a alguien realizando una acción motriz que está dentro de nuestro repertorio, nuestro cerebro crea las condiciones para simular la acción visionada, resultando una metodología útil de investigación puesto que permite reflejar el conocimiento que se tiene sobre el acto motor. Este enfoque, incluido dentro de las técnicas empleadas en psicología del deporte como "visualización", ofrece una perspectiva fructífera para interpretar la importancia esencial de los vínculos establecidos entre la representación mental del movimiento y la acción motriz (Moran, 2009).

Una vez presentados los principales aspectos conceptuales en los que se basa esta investigación y detectada la ausencia de trabajos sobre el conocimiento de base de los jugadores con DIL en el FS, el objetivo principal de este estudio se centra en construir y validar una herramienta que permita conocer, de forma fiable, el conocimiento de base de jugadores con DIL en aspectos de táctica ofensiva en FS. Debido a las limitaciones de acceso a la población de estudio, por el reducido número de practicantes, esta investigación pretende ser un primer estudio piloto sobre las propiedades del instrumento.

\section{MÉTODO}

El diseño de la investigación combina la metodología cuantitativa con la cualitativa a través de la realización de una entrevista grupal. Consideramos, como manifiestan Castañer, Camerino, \& Anguera (2013), que esta metodología se está afianzando en la última década en consonancia con la necesidad de conjugar ambas orientaciones en planteamientos más integrados en la investigación de la motricidad humana.

\section{Participantes:}

Para el reclutamiento muestral, se debe enfatizar la complejidad del acceso a la muestra con las características pretendidas. El tamaño muestral para este estudio piloto está constituido por 68 sujetos con $M_{\text {edad }}=27$; $S D_{\text {edad }}=9,06$ y

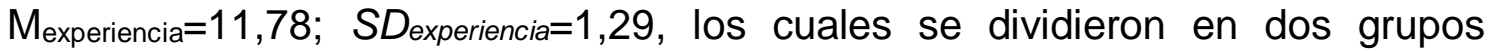
teniendo en cuenta el factor de discapacidad (sí/no). Todos los jugadores participantes en el estudio y pertenecientes al grupo con DIL, tenían el informe que certificaba su nivel. Grupo A: formado por 34 jugadores de FS con DIL $\left(M_{\text {edad }}=24,5 ; S D_{\text {edad }}=7,11\right.$ y Mexperiencia $=11,09$; $S D_{\text {experiencia }}=0,93$ ) que participan en las ligas de División de Honor y Primera División organizadas por la Federación Madrileña de Deportes para Discapacitados Intelectuales (FEMADDI). Grupo B: constituido por 34 jugadores de FS $\sin$ DIL (Medad=29,2; $S D_{\text {edad }}=10,25$ y Mexperiencia $\left.=11,52 ; S D_{\text {experiencia }}=1,16\right)$.

El grupo A fue seleccionado dentro del colectivo diana con DI que practica FS. Todos sus jugadores fueron diagnosticados como personas con DIL. El grupo B 
se formó de manera incidental. La participación fue voluntaria y consentida, y se respetaron todos los términos éticos de la Declaración de Helsinki (World Medical Association, 2013), contando con la aprobación del Comité de Ética.

\section{Instrumento}

La metodología empleada para la investigación se basa en la recogida de datos, a través un instrumento creado ad hoc, sobre la evaluación para validar una herramienta que permita la evaluación del conocimiento de base en la toma de decisiones de la fase ofensiva para jugadores de FS con DIL. Se ha seleccionado la fase ofensiva porque, al tratarse de un estudio piloto, se ha pretendido limitar la complejidad táctica aumentando la profundidad en el conocimiento sobre estos principios del juego por parte de los jugadores con DIL.

La elaboración del instrumento parte de una revisión de la literatura científica sobre acciones técnico-tácticas en la fase de ataque para las situaciones de juego en el FS (Gallego-Jiménez, 2013; Hermans \& Engler, 2010), empleando una metodología similar a la planteada por De la Vega (2003), en la realización de su tesis doctoral sobre comprensión táctica en fútbol, y por De la Vega et al. (2008) Se obtuvieron 26 posibles acciones según las dos variables que proponen Hernández-Moreno (2005) y Lasierra-Aguilá \& Escudero-Pereira (1993): jugador con balón en fase de ataque (18 acciones), jugador sin balón en fase de ataque (8 acciones). Estas acciones fueron ratificadas por dos especialistas del FS, con 20 años de experiencia en el ámbito del entrenamiento para personas con DI (Universidad Autónoma de Madrid y Fundación A LA PAR), como aspectos a trabajar en la planificación de entrenamientos para este colectivo con discapacidad.

Para profundizar en la validez criterial y cumplir con los principios establecidos en la realización del proceso de validación de un contenido (Skjong \& Wentworth, 2001), se contactó con 14 profesionales-expertos, con más de 10 años de experiencia en entrenamientos del FS en personas con DI (Mexperiencia $=12,42$; $\left.S D_{\text {experiencia }}=2,10\right)$. Se les solicitó que puntuaran las 26 acciones técnico-tácticas en función de la dificultad en la comprensión, que ellos consideraban, que tendrían para los jugadores con DIL. Esta puntuación se llevó a cabo mediante una encuesta de tipo escala Likert (de 1-mayor dificultad a 5-menor dificultad), para limitar el número de acciones a las más comprensibles por parte de este colectivo. Los expertos señalaron con el valor máximo (5) un total de cuatro acciones: apoyo, desmarque, pase y remate.

Tras la selección de estas cuatro acciones, se representaron gráficamente 24 Ítems (IT) con tres jugadas/opciones posibles de respuestas (A, B o C) (De la Vega, 2003). Para la inclusión de los IT, en función de su pertinencia del contenido y del nivel de comprensión, se siguieron los criterios de rigurosidad de Cohen \& Swerdlik (2001) y Voutilainen \& Liukkonen (1995), reduciendo los IT a 12 al superar el $80 \%$ de los profesionales-expertos que estuvieron de acuerdo con la pertinencia de cada IT. 
Debido a la gran simbología de la representación gráfica y las dificultades que presentan los jugadores con DIL en el procesamiento de la información, el diseño del instrumento se optó por realizar una presentación de forma audiovisual por cada uno de los 12 IT (Bailey, Willner, \& Dymond, 2011; Fisher, Bailey, \& Willner, 2012).

La cámara de vídeo utilizada fue SMX-F40BN (Samsung manual 65xintellzoom), con un sensor CCD de 1/6" con $680 \mathrm{~K}$, zoom óptico de 52x, formato de grabación H.264 y resolución 720x480. Posteriormente, se realizó la maquetación con el programa "After Effects" para su posterior incorporación en un formato audiovisual (se proporciona enlace del cuestionario final). Los participantes en este proceso se seleccionaron de forma incidental, por motivos de disponibilidad y con el criterio de 15 años de experiencia en FS.

\section{https://m.youtube.com/watch?feature=youtu.be\&v=1H8EXW9qz7Y}

Tras diseñar el instrumento, se contó con la colaboración de 28 entrenadores de FS con nivel uno o superior según la Federación Madrileña de FS. Este grupo de profesionales-expertos con $M_{\text {edad }}=33,25 ; S D_{\text {edad }}=12,33$ y $M_{\text {experiencia }}=12,25$; $S D_{\text {experiencia }}=8,51$, establecieron un juicio de valor sobre las jugadas/opciones (A, B o C) de cada IT, dando una puntuación de cero a la opción menos correcta, uno a la opción aceptable y dos a la opción más correcta, bajo los parámetros de una evaluación criterial de jueces para determinar una puntuación estándar (Jornet-Melía \& González-Shuch, 2009). La interpretación de las puntuaciones de este grupo, permitieron asignar un puntaje medio por cada jugada/opción (expuestos en resultados).

Para finalizar con este proceso de construcción de una herramienta, se procedió a un estudio piloto con tres personas con DIL para comprobar si eran capaces de comprender cada una de las jugadas/opciones que componían los 12 IT del audio-vídeo y generar una hoja test que pudieran marcar con una X la opción elegida. Los resultados fueron positivos, a la hora de concretar una respuesta, sin que mostraran o expresaran dificultad a la hora de ejecutar la prueba (CarbóCarreté, Giné, \& Guardia-Olmos, 2013).

\section{Procedimiento}

A los jugadores se les presentó la herramienta, a través de condiciones programadas en el laboratorio (French \& Thomas, 1987), en un espacio habilitado en las instalaciones deportivas. Cada jugador tenía la hoja test e iba contestando según visualizaba el IT, con 5 segundos aproximadamente entre cada IT. No se permitió que coincidieran más de tres jugadores juntos. La recogida de datos fue realizada a mitad de temporada, utilizando sesiones de entrenamiento.

Antes de comenzar con las respuestas, los jugadores fueron instruidos para visualizar las tres jugadas/opciones de un IT y por último seleccionar una respuesta. Además, se les informó sobre la intención de la herramienta y su desarrollo: 
"El vídeo-cuestionario está formado por acciones de ataque, basadas en los aspectos fundamentales de la fase ofensiva. En cada una de las acciones que vas a ver en la pantalla debes elegir (tomar una decisión) una de las tres posibilidades A, B o C. Estas acciones se muestran, a través de una filmación de diferentes jugadas. Tú eres el jugador que ataca y está marcado con una flecha encima".

\section{Análisis estadístico}

El análisis estadístico de los datos se realizó con el programa SPSS 22.0 para Windows (IBM-SPSS, 2013). La estadística descriptiva de las respuestas por el grupo de 28 entrenadores permitió obtener una puntuación $M$ por cada posible jugada/opción ( $A, B$ o $C$ ). Este valor numérico se tomó como referencia en las respuestas seleccionadas por los jugadores de la muestra.

Para conocer la fiabilidad de la herramienta, a través de las respuestas de la muestra $(N=68)$, se realizó la estimación de los coeficientes: Alfa de Cronbach $(\alpha)$, Coeficiente Omega ( $\omega$ ) (Elosua-Oliden \& Zumbo, 2008). Mediante el Coeficiente de Correlación Intraclase (ICC) se calculó la consistencia con la finalidad de evaluar el grado de acuerdo o concordancia intraclase, propuesto por Landis \& Koch (1977).

La estabilidad o repetitividad de las respuestas se evaluó con el método testretest (Cuevas, García-López, \& Contreras, 2015; Moreno, Moreno, GarcíaGonzález, Gil, \& Del Villar, 2010; Ruiz-Pérez \& Graupera, 2005), mediante el que se comparan las respuestas de un mismo grupo pasados más de 10 días. Este grupo $(N=20)$ se constituyó a partir de la muestra. Se compararon mediante el método paramétrico de la $t$ de Student para muestras pareadas con el fin de observar la existencia de diferencias significativas en su nivel de significación (p).

Con la intención de aportar información específica de ambos grupos, se añade un análisis factorial con el método de extracción de los componentes principales $(\mathrm{CP})$, con el que se construyó el valor Factor (FAC) como puntuación media total de los 12 IT para cada jugador (Liu, Kuang, Gong, \& Hou, 2003). Este FAC permite comparar y observar la existencia o no de diferencias significativas entre los dos grupos.

\section{Análisis cualitativo}

Debido a que los investigadores consideraron necesario proporcionar una mejor comprensión del proceso de implementación y validación de la prueba, para que fuese compatible con la perspectiva del modelo social (Oliver, 1992) actualmente vigente, se recogieron las percepciones de los entrenadores con experiencia en entrenamientos de FS en personas con DI que participaron en el proceso, utilizando para ello una entrevista grupal, lo que McKernan (1999) denomina seminario, que permitiese un debate en profundidad con tres expertos y relacionado con el objeto de estudio. En efecto, como se verá posteriormente en 
el análisis, la conversación giró en torno a la utilidad, validez y adecuación de la prueba, utilizando situaciones reales y analizando la dificultad de la misma, utilizando un contraste con la información obtenida con el análisis estadístico y una triangulación entre los expertos participantes.

\section{RESULTADOS}

Las respuestas ofrecidas por los entrenadores, de cada una de las tres jugadas/opciones (A, B o C) de los 12 IT, a través de la aplicación de estadística descriptiva sobre las variables cuantitativas, permiten obtener unos valores numéricos (puntuaciones estándar) con la $M$ (tabla 2).

Tabla 2. Análisis descriptivo de las respuestas de los entrenadores.

\begin{tabular}{|c|c|c|c|c|}
\hline$I T$ & Jugadas/opciones & $\bar{N}$ & $M$ & $S D$ \\
\hline \multirow[t]{3}{*}{ IT1 } & $A$ & 28 & 1,21 & 0,83 \\
\hline & B & 28 & 0,32 & 0,55 \\
\hline & $\mathrm{C}$ & 28 & 1,46 & 0,58 \\
\hline \multirow[t]{3}{*}{ IT2 } & A & 28 & 0,32 & 0,61 \\
\hline & B & 28 & 0,86 & 0,59 \\
\hline & $\mathrm{C}$ & 28 & 1,82 & 0,39 \\
\hline \multirow[t]{3}{*}{ IT3 } & $A$ & 28 & 0,75 & 0,75 \\
\hline & B & 28 & 0,79 & 0,79 \\
\hline & C & 28 & 1,46 & 0,74 \\
\hline \multirow[t]{3}{*}{ IT4 } & $A$ & 28 & 1,43 & 0,74 \\
\hline & B & 28 & 0,89 & 0,83 \\
\hline & C & 28 & 0,68 & 0,72 \\
\hline \multirow[t]{3}{*}{ IT5 } & $A$ & 28 & 1,43 & 0,63 \\
\hline & B & 28 & 0,68 & 0,82 \\
\hline & $\mathrm{C}$ & 28 & 0,89 & 0,83 \\
\hline \multirow[t]{3}{*}{ IT6 } & A & 28 & 0,82 & 0,86 \\
\hline & B & 28 & 1,5 & 0,75 \\
\hline & C & 28 & 0,68 & 0,61 \\
\hline \multirow[t]{3}{*}{ IT7 } & $A$ & 28 & 1,68 & 0,48 \\
\hline & B & 28 & 0,00 & 0,00 \\
\hline & $\mathrm{C}$ & 28 & 1,32 & 0,48 \\
\hline \multirow[t]{3}{*}{ IT8 } & A & 28 & 1,32 & 0,72 \\
\hline & B & 28 & 0,29 & 0,66 \\
\hline & C & 28 & 1,39 & 0,57 \\
\hline \multirow[t]{3}{*}{ IT9 } & $A$ & 28 & 0,29 & 0,6 \\
\hline & B & 28 & 1,5 & 0,64 \\
\hline & C & 28 & 1,21 & 0,69 \\
\hline \multirow[t]{3}{*}{ IT10 } & $A$ & 28 & 1,75 & 0,44 \\
\hline & B & 28 & 0,61 & 0,79 \\
\hline & C & 28 & 0,64 & 0,62 \\
\hline \multirow[t]{3}{*}{ IT11 } & A & 28 & 0,89 & 0,74 \\
\hline & B & 28 & 0,5 & 0,75 \\
\hline & C & 28 & 1,61 & 0,57 \\
\hline \multirow[t]{3}{*}{ IT12 } & $A$ & 28 & 1,71 & 0,46 \\
\hline & $B$ & 28 & 0,00 & 0,26 \\
\hline & C & 28 & 1,0 & 0,57 \\
\hline
\end{tabular}


Posteriormente, tras obtener las respuestas de los 68 sujetos de la muestra, se realizó una extrapolación de los valores $M$ por cada jugada/opción (A, B o C). De esta forma, se adjudicó un valor numérico a las decisiones tomadas por la muestra, al elegir una de las jugadas/opciones que visualizan con el vídeocuestionario en cada uno de los 12 IT.

Al tomar de referencia esas puntuaciones, se dio paso al análisis del índice de fiabilidad de la herramienta con $\alpha=0,64$, con un valor por debajo de lo aceptable $(0,7)$ propuesto por George \& Mallery $(2003)$. A su vez, este resultado muestra una fiabilidad adecuada para estudios exploratorios, como defienden Fisher, Matthews \& Gibbons (2016) y Nunnally \& Bernstein (1978).

Pero este coeficiente de fiabilidad parte de la ponderación de las covariancias de los IT y de la cantidad de IT, es decir, la proporción de la variancia total del instrumento y del número de IT.

$$
\propto=\frac{n}{n-1}\left(1-\frac{\sum_{i} V_{i}}{V_{t}}\right)
$$

El análisis de $\omega$ dio un valor de 0,74 , dentro de los valores aceptables de confiabilidad, a través de la fórmula expuesta por McDonald (1999):

$$
\omega=\frac{\left(\sum_{i=1}^{n} \lambda_{j}\right)^{2}}{\left(\sum_{i=1}^{n} \lambda_{j}\right)^{2}+\sum_{i=1}^{n} \operatorname{var}(e)_{i i}}
$$

La consistencia se evaluó mediante el ICC, mostrando una consistencia notable o satisfactoria al ser un $\mathrm{ICC}=0,64$, dentro del rango $[0,6-0,8]$ propuesto por Landis \& Koch (1977).

Con el método test-retest, no se encontraron diferencias significativas con niveles de confianza del 95\% en los 12 IT (tabla 2).

Tabla 3. Prueba de $t$ de Student de las respuestas del grupo test- retest

\begin{tabular}{lllllllllllll}
\hline $\mathbf{N}=20$ & IT1 & IT2 & IT3 & IT4 & IT5 & IT6 & IT7 & IT8 & IT9 & IT10 & IT11 & IT12 \\
\hline $\boldsymbol{p}$ & 0,15 & 0,46 & 0,33 & 0,33 & 0,11 & 0,15 & 0,06 & 0,70 & 0,35 & 0,97 & 0,88 & 0,40 \\
\hline
\end{tabular}

Tras aportar los resultados para la validez y fiabilidad de la herramienta, se construyó el valor FAC, mediante un análisis factorial con el método de extracción de los CP. La reducción de los datos permitió explicar la mayor cantidad de información posible para los 12 IT del cuestionario, y se objetivó que los pesos factoriales del CP1 proporcionaban el mayor porcentaje de explicación del modelo (Figura 1) (tabla 3). 
Figura 1: Representación gráfica de sedimentación.

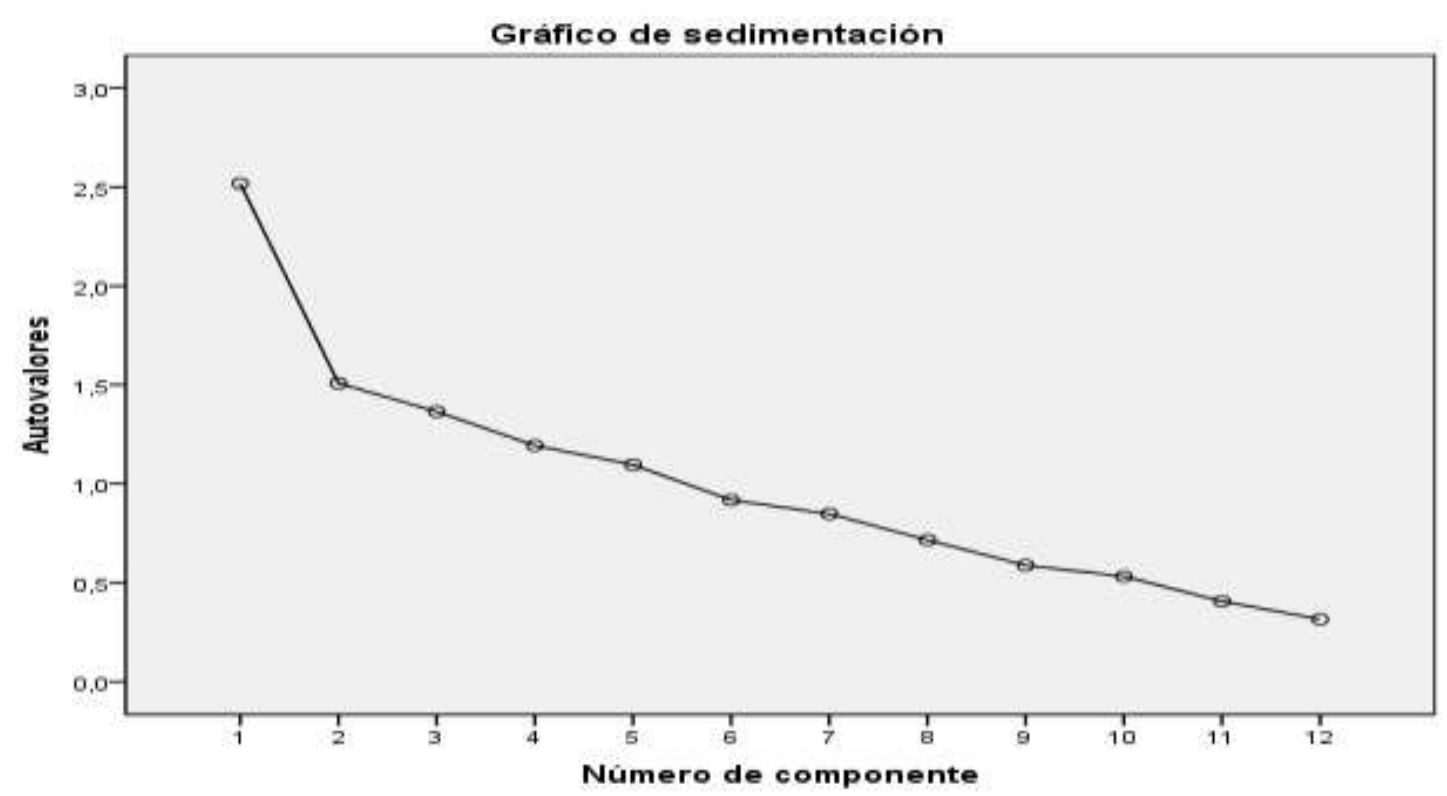

Tabla 4. Cargas factoriales del CP1.

Matriz de componentes

\begin{tabular}{lllllllllllll} 
& IT1 & IT2 & IT3 & IT4 & IT5 & IT6 & IT7 & IT8 & IT9 & IT10 & IT11 & IT12 \\
CP1 & 0,514 & 0,423 & 0,293 & 0,030 & 0,338 & 0,410 & 0,655 & 0,577 & 0,476 & 0,628 & 0,309 & 0,478 \\
\hline
\end{tabular}

Estas cargas factoriales del CP1 se emplearon para el cálculo de los coeficientes de cada IT (tabla 4), que da lugar a la construcción del FAC mediante una fórmula logarítmica:

$$
X_{i j}=a_{i 1} \cdot Z_{1 j}+a_{i k} \cdot Z_{k j}=\sum_{s=1}^{k} a_{i s} \cdot Z_{s k}
$$

Tabla 5. Coeficientes para el cálculo de la puntuación del FAC.

\begin{tabular}{lllllllllllll}
\hline \multicolumn{10}{c}{ Matriz coeficientes puntuaciones componentes } \\
\hline & IT1 & IT2 & IT3 & IT4 & IT5 & IT6 & IT7 & IT8 & IT9 & IT10 & IT11 & IT12 \\
CP1 & 0,204 & 0,168 & 0,116 & 0,012 & 0,134 & 0,163 & 0,260 & 0,229 & 0,189 & 0,249 & 0,123 & 0,190 \\
\hline
\end{tabular}

La fórmula logarítmica del propio modelo de la construcción del FAC, genera un valor numérico global de la totalidad de las respuestas de la muestra. El valor $M$ del FAC fue 0 , con una desviación típica $(S D)$ de 1 . Esto permite asumir que la puntuación del $F A C=0$ es considerada aceptable mientras que los valores del $\mathrm{FAC}<0$ serán menos correctos y valores del $\mathrm{FAC}>0$ serán más correctos.

Con respecto a los dos grupos tratados en la investigación, las respuestas obtenidas por la muestra responden al siguiente patrón respecto a la puntuación $M$ del FAC expuesto en la tabla 5. 
Tabla 6. M del FAC en todos los grupos.

\begin{tabular}{lll}
\hline & Grupo A & Grupo B \\
\hline M FAC & $-0,364$ & 0,364 \\
\hline
\end{tabular}

Como anteriormente se ha comentado, para una mejor comprensión del proceso, se complementa el análisis estadístico con el análisis cualitativo aportando la información que se obtuvo en las preguntas más representativas de la entrevista grupal:

Pregunta: ¿Una prueba como esta que presenta varias alternativas puede resultar, desde vuestro punto de vista, útil para presentar en una etapa formativa los principios básicos de ataque y esas alternativas como conocimiento de base? (Tutor2-UCJ-28'.45").

Respuestas: "Yo creo que el deportista con DIL seguramente sí llegue a concebir las imágenes del cuestionario. Generalmente el jugador con DIL va a dar la respuesta que a él siempre le ha dado mejor experiencia, porque es la que tiene interiorizada y la que primero le va a salir. Considero que tienen poca intención de explorar respuestas alternativas" (Part3-UCJ-29'.07').

Esta manifestación es reforzada por el participante 2 al considerar que pueden aprenderla siempre que se utilice en situación real de juego y con el suficiente tiempo:

"Es verdad que, si de alguna manera se puede cambiar (la conducta), la idea del vídeo, a base de trabajarlo y trabajarlo sí que puede ser útil. A mí es la mejor prueba que se me puede ocurrir" (Part2-UCJ-30'.21').

En la siguiente manifestación, podemos observar que el participante 1 también está de acuerdo con la validez de la prueba, destacando las alternativas que ofrece:

"Sí, va a aprender otra manera pero que le sirve también para decidir dentro de las posibilidades a elegir" (Part1-UCJ-30'.32").

Posteriormente, se pidió la opinión a los expertos sobre la adecuación de la herramienta para la edad y a la experiencia por parte de los jugadores con DIL.

Pregunta 6: ¿Habría algún referente relacionado con el nivel de experiencia o sobre las categorías de competición, donde la utilidad del cuestionario fuese adecuado y se pudiese aprovechar al máximo? (Tutor 2-UCJ-33'.39”).

Respuestas: "Yo no hablaría de edades, no es una variable a tener en cuenta en jugadores con DIL" (Part2-UCJ-34'.03"'). 
Se puede observar que para el experto 2 la edad no sería un referente en jugadores con DIL Sin embargo, para el experto 3 la edad estaría sobre los 1618 años ya que considera que a esa edad podrá tener experiencia para comprender la intencionalidad de la prueba. Para este experto la dificultad más importante está en la aplicación del conocimiento en las situaciones:

Respuesta. "Una edad que yo creo que puede estar entre los 16-18 años, que sí es proclive a que él empiece a comprender cuál es la intención de la prueba. Desde mi punto de vista, uno de los hándicaps es que el jugador comprenda lo que les estoy presentando y que sirve para mejorar su juego. El problema surge llevarlo a la práctica cuando estoy jugando" (Part3-UCJ-34'.12").

En este sentido, el Experto 1 pone el énfasis en la necesidad de darles tiempo para el aprendizaje, al menos un curso escolar.

Respuesta. "Yo creo que debería de tener una formación en alguna escuela deportiva por lo menos de un curso escolar para comprender la lógica de juego" (Part1-UCJ-35'.25").

\section{DISCUSIÓN}

La herramienta propuesta en esta investigación se basa en la utilización de situaciones simuladas y controladas, a través de la cual se ha conseguido evaluar el conocimiento de base en jugadores con DIL mediante estructuras cognitivas que permiten tomar decisiones que involucran situaciones tácticas del juego, como son las acciones de apoyo, desmarque, pase y tiro.

En la validación de la herramienta, se empleó un modelo mixto en el cual se mezclan datos cuantitativos y cualitativos, para dar mayor solidez a los resultados. La utilidad de este método se ha ido implantando durante los últimos años dentro del campo de la Ciencia de la Actividad Física y del Deporte (Castañer, Camerino, \& Anguera, 2013). A su vez, Johnson, Onwuegbuzie \& Turner (2007) promueven el desarrollo de estos modelos mixtos para la comprensión y corroboración en la amplitud y profundidad de los propósitos en una investigación.

El uso de a asume un modelo de medida que pondera las varianzas de error de los IT de igual manera (tau equivalencia), interpretando el carácter continuo de sus variables cuando son estrictamente paralelos en la matriz de correlaciones (López-Pina, Sánchez-Meca, \& López-López, 2012; Raykov \& Marcoulides, 2015; Cronbach, 1951).

Esta medición de fiabilidad es discutida y cuestionada, al producirse violaciones en el supuesto de tau-equivalencia de una escala que produzca la misma sensibilidad en todos los IT, al ser poco realista. Por ese motivo, es necesario aplicar otras medidas de fiabilidad que permitan estimar la consistencia interna, asumiendo los propios errores correlacionales de cada IT, a través de un 
constructo o factor. El error es único para cada IT y está incorrelacionado con la puntuación factorial y también con los errores de los otros IT (Deng \& Chan, 2017; Jöreskog, 1971; Zinbarg, Revelle, Yovel, \& Li, 2005; Viladrich, AnguloBrunet, \& Doval, 2017).

Se considera $\omega$ una alternativa práctica a a para estimar la fiabilidad en la consistencia interna en un modelo factorial, a través de los pesos factoriales $\left(\lambda_{j}\right)$ por cada IT, partiendo de un modelo de medidas congenéricas que permite diferenciar valores de discriminación entre los IT (Revelle \& Zinbarg, 2009; McDonald, 1999; Dunn, Baguley, \& Brunsden, 2014; Zhang \& Yuan, 2016; Raykov, 1997).

La herramienta formada por 12 IT presenta un $\omega$ de 0.74 , el cual se sitúa con valores óptimos de consistencia interna; así mismo, las dimensiones arrojan un coeficiente por encima de 0.70 afirmando su confiabilidad (Bruner \& Benson, 2018; Iturbide-Luquin \& Elosua-Oliden, 2017; Viciana, Mayorga-Vega, GuijarroRomero, \& Martínez-Baena, 2017; Viladrich, Angulo-Brunet, \& Doval, 2017).

Por otro lado, los requisitos que debe cumplir un instrumento de medición son: validez, confiabilidad y estabilidad. Para demostrar estas premisas, se aporta la prueba cuantitativa test-retest que confirmó la estabilidad de las respuestas de los IT.

Mediante una prueba cualitativa, la totalidad de participantes en el debate afirmaron que estos jugadores serían capaces de comprender la intención de la herramienta. Así mismo, los expertos avalaron el hecho de que dicha herramienta podría ser un buen instrumento de apoyo en el proceso de entrenamiento de la táctica del FS, al mostrar a los jugadores diferentes opciones válidas ante una situación de juego específica. Los expertos valoraron positivamente la herramienta como un posible método para evaluar el nivel de conocimiento de base los jugadores con DIL.

Las respuestas ofrecidas por el grupo A se encuentran por debajo de lo que se considera aceptable en la puntuación del FAC. Este hecho muestra que al comparar la $M$ con el grupo $\mathrm{B}$, sí presentan grandes diferencias en su conocimiento de base táctico declarativo que influye en su toma de decisiones. Este resultado se ratifica en los trabajos realizados por Pinilla et al. (2016), Pinilla et al. (2017), Van Biesen et al. (2014) y Van Biesen et al. (2016) donde los jugadores con DIL tienen decisiones más erróneas al compararlos con los jugadores sin DIL, a pesar de practicar el mismo deporte.

\section{CONCLUSIONES}

La herramienta propuesta y creada durante este período de investigación cumple los criterios de validez, fiabilidad y estabilidad para la toma de datos, elaboración de análisis y obtención de resultados. La herramienta permite establecer el conocimiento de base en acciones tácticas ofensivas para jugadores de FS con DIL. 
El análisis de la información obtenida refleja que los jugadores con DIL son capaces de tomar decisiones a pesar de sus dificultades cognitivas. En la comparación de las respuestas entre los jugadores con y sin DIL, se obtuvieron diferencias en relación sobre el conocimiento de base en situaciones de ataque en FS. Las respuestas proporcionadas en el grupo con DIL, estuvieron por debajo de lo considerado aceptable $(\mathrm{FAC}=0)$.

Se considera que este artículo forma parte de un inicio en la investigación referida a la toma de decisiones en personas con DI desde el ámbito de las actividades físicas. Es por ello, que se considera pertinente y necesario la colaboración en esta línea de investigación para contribuir a la innovación y progreso en la atención a las personas con discapacidad desde las actividades físicas en sus distintas posibilidades.

\section{REFRENCIAS BIBLIOGRÁFICAS}

Abernethy, B., Thomas, K. T., \& Thomas, J. T. (1993). Strategies for improving understanding of motor expertise. En J. L. Starkes, \& F. Allard, Cognitive issues in motor expertise (págs. 317-356). Amsterdam: Elsevier.

Allard, F., Deakin, J., Parker, S., \& Rodgers, W. (1993). Declarative Knowledge in skilled motor performance: by product or constituent? En J. L. Starkes, \& F. Allard, Cognitive issues in motor expertise (págs. 95-108). Amsterdam: Elsevier.

Anderson, J. R. (1987). Skill acquisition: compilation of weak--method problem solutions. Psychological Review, 94(2), 192-210. DOI:10.1037/0033295X.94.2.192

Araújo, D., \& Davids, K. (2011). What exactly is acquired during skill acquisition? Journal of Consciousness Studies, 18(3-4), 7-23.

Bailey, R., Willner, P., \& Dymond, S. (2011). A visual aid to decision-making for people with intellectual disabilities. Research in Developmental Disabilities, 32(1), 37-46. DOI: 10.1016/j.ridd.2010.08.008

Bruner, M. W., \& Benson, A. J. (2018). Evaluating the psychometric properties of the Social Identity Questionnaire for Sport (SIQS). Psychology of Sport and Exercise, 35, 181-188. https://doi.org/10.1016/j.psychsport.2017.12.006

Calvo-Merino, B., Glaser, D. E., Grèzes, J., Passingham, R. E., \& Haggard, P. (2004). Action observation and acquired motor skills: an FMRI study with expert dancers. Cerebral cortex, 15(8), 1243-1249. https://doi.org/10.1093/cercor/bhi007

Carbó-Carreté, M., Giné, C., \& Guardia-Olmos, J. (2013). Estudio piloto para la elaboración de un cuestionario para la evaluación de hábitos y necesidades de apoyo para la actividad física en personas con discapacidad intelectual. Revista Iberoamericana de Psicología del Ejercicio y el Deporte, 8(1), 193-208.

Castañer, M., Camerino, O., \& Anguera, M. T. (2013). Métodos mixtos en la investigación de las ciencias de la actividad física y el deporte. Apunts. Educación Física y Deportes, 31-36. 
Cohen, R., \& Swerdlik, M. (2001). Pruebas y Evaluación Psicológica. Introducción a las pruebas y a la Medición (4th edición). México: McGraw Hill.

Cronbach, L. J. (1951). Coefficient alpha and the internal structure of test. Psychometrika, 16(3), 297-334. https://doi.org/10.1007/BF02310555

Cuevas, R., García-López, L. M., \& Contreras, O. (2015). Influencia del modelo de Educación Deportiva en las necesidades psicológicas básicas. Cuadernos de Psicología del Deporte, 15(2), 155-162. http://dx.doi.org/10.4321/S1578-84232015000200017.

Davids, K., Renshaw, I., Pinder, R. A., Araújo, D., \& Vilar, L. (2012). Principles of motor learning in ecological dynamics. A comment on functions of learning and the acquisition of motor skills (with reference to sport). The Open Sports Sciences Journal, 5, 113-117. DOI: 10.2174/1875399X0120501013

De la Vega, R. (2003). Desarrollo del metaconocimiento táctico y comprensión del juego. Un enfoque constructivista aplicado al fútbol (tesis doctoral). Madrid: Universidad Autónoma.

De la Vega, R., Del Valle, S., Maldonado, A., \& Moreno, A. (2008). Pensamiento y acción en el deporte. Sevilla: Wanceulen.

Deng, L., \& Chan, W. (2017). Testing the difference between reliability coefficients alpha and omega. Educational and Psychological Measurement, 77(2), 185-203. DOI:10.1177/0013164416658325

Dunn, T. J., Baguley, T., \& Brunsden, V. (2014). From alpha to omega: A practical solution to the pervasive problem of internal consistency estimation. British Journal of Psychology, 105(3), 399-412. DOI: 10.1111/j.13652788.2009.01228.x.

Elosua-Oliden, P., \& Zumbo, B. (2008). Coeficientes de fiabilidad para escalas de respuesta categórica ordenada. Psicothema, 20(4), 896-901.

Elwyn, G., \& Miron-Shatz, T. (2010). Deliberation before determination: the definition and evaluation of good decision making. Health Expectations, 13(2), 139-147. DOI: 10.1111/j.1369-7625.2009.00572.x

Fernández, C., Moreno, A., Gil, A., Claver, F., \& Moreno, M. P. (2014). Estudio del conocimiento procedimental, experiencia y rendimiento, en jóvenes jugadores de voleibol. Retos. Nuevas tendencias en Educación Física, Deporte y Recreación(25), 13-16.

Fisher, G. G., Matthews, R. A., \& Gibbons, A. M. (2016). Developing and investigating the use of single-item measures in organizational research. Journal of Occupational Health Psychology, 21(1), 3-23. https://doi.org/10.1037/a0039139

Fisher, Z., Bailey, R., \& Willner, P. (2012). Practical aspects of a visual aid to decision making. Journal of Intellectual Disability Research, 56(6), 588599. https://doi.org/10.1111/j.1365-2788.2011.01498.x.

Franciosi, E., Gallotta, M. C., Baldari, C., \& Emerenziani, G. P. (2012). Basketball ability testing and category for players with mental retardation: 8-month training effect. The Journal of Strength and Conditioning Research, 15241531. DOI: 10.1519/JSC.0b013e318236d0a4

French, K. E., \& Thomas, J. R. (1987). The Relation off Knowledge Development to Children's Basketball Performance. Journal of sport psychology, 9(1), 15-32. DOI: 10.1123/jsp.9.1.15. 
Gallego-Jiménez, A. L. (2013). La táctica ofensiva en el futsal : importancia de la visión en el desarrollo del juego. Murcia: A.L. Gallego.

George, D., \& Mallery, P. (2003). Spss for Windows step by step: A Simple Guide and Reference. 11.0 Update(4. ${ }^{a}$ ed.). Boston: Allyn \& Bacon.

Gréhaigne, J. F., Wallian, N., \& Godbout, P. (2005). Tactical-decision learning model and students' practices. Physical Education and Sport Pedagogy, 10(3), 255-269. DOI: 10.1080/17408980500340869.

Guidetti, L., Franciosi, E., Emerenziani, G. P., Gallotta, M. C., \& Baldari, C. (2009). Assessing basketball ability in players with mental. British Journal of Sports Medicine, 43, 208-2012. DOI: 10.1136/bjsm.2006.034918.

Hermans, V., \& Engler, R. (2010). Futsal: Technique, Tactics, Training. UK: Meyer \& Meyer Verlag.

Hernández-Moreno, J. (2005). Análisis de las estructuras del juego deportivo. Barcelona: Inde.

IBM-SPSS. (2013). SPSS Statistics 22.0 Command Syntax Reference. SPSS Inc.

Iturbide-Luquin, L. M., \& Elosua-Oliden, P. (2017). Los valores asociados al deporte: análisis y evaluación de la deportividad. Revista de Psicodidáctica, 22(1), 29-36. https://doi.org/10.1016/S11361034(17)30041-2

Johnson, R. B., Onwuegbuzie, A. J., \& Turner, L. A. (2007). Toward a Definition of Mixed Methods Research. Journal of Mixed Methods Research, 112133. DOI: $10.1177 / 1558689806298224$.

Jöreskog, K. G. (1971). Statistical analysis of sets of congeneric tests. Psychometrika, 36(2), 109-133. http://dx.doi.org/10.1007/BF02291393

Jornet-Melía, J. M., \& González-Shuch, J. (2009). Evaluación criterial: determinación de estándares de interpretación (EE) para pruebas de rendimiento educativo. Estudios sobre Educación, 16, 103-123.

Kelso, J. S. (2012). Multi-stability and meta- stability: understanding dynamic coordination in the brain. Philosophical Transactions of the Royal Society B: Biological Sciences, 367(1591), 906-918. https://doi.org/10.1098/rstb.2011.0351

Landis, J. R., \& Koch, G. G. (1977). The measurement of observer agreement for categorical data. Biometrics, 33(1), 159-174. DOI: 10.2307/2529310.

Lasierra-Aguilá, G., \& Escudero-Pereira, P. (1993). Observación y evaluación en los deportes de cooperación-oposición: en busca de sus aspectos distintivos. Apunts, 86-105.

Lim, C. P., \& Jain, L. C. (2010). Advances in intelligent decision making. En L. C. Jain, \& C. P. Lim, Handbook on decision making. Vol 1. Techniques and applications (págs. 3-28). Springer.

Liu, R., Kuang, K., Gong, Q., \& Hou, X. (2003). Principal component regression analysis with SPSS. Computer Methods and Programs in Biomedicine, 71(2), 141-147. https://doi.org/10.1016/S0169-2607(02)00058-5

López-Pina, J. A., Sánchez-Meca, J., \& López-López, J. A. (2012). Métodos para promediar coeficientes alfa en los estudios de generalización de la fiabilidad. Psicothema, 24(1), 161-166.

López-Ros, V. (2011). El Pensamiento Táctico y su desarrollo. En V. López-Ros, \& J. Sargatal, La Táctica individual en los deportes de equipo (págs. 7593). Girona: Universidad de Girona. 
MacMahon, C., \& McPherson, S. L. (2009). Knowledge base as a mechanism for perceptual-cognitive tasks: Skills is in the details! International Journal of Sport Psychology, 40, 565-579.

Matias, C. J., \& Greco, P. J. (2010). Cognição \& ação nos jogos esportivos coletivos: Cognition and Action in team ball sports. Ciências \& Cognição, 15(1), 252-271.

McDonald, R. P. (1999). Test theory: A unified treatment. Mahwah: Lawrence Erlbaum.

McKernan, J. (1999). Investigación - acción y currículo. Madrid: Morata.

McPherson, S. L., \& Kernodle, M. (2007). Mapping two new points on the tennis expertise continuum: tactical skills of adult advanced beginners and entrylevel professionals during competition. Journal of Sports Sciences, 25(8), 945-959. https://doi.org/10.1080/02640410600908035.

Meilán, J. J., Salgado, V. M., Arana, J. M., Carro, J., \& Jenaro, C. (2008). Entrenamiento cognitivo y mejora de la memoria prospectiva en jóvenes con retraso mental leve. Revista de Investigación Educativa, 26(1), 227245.

Moran, A. (2009). Cognitive psychology in sport: Progress and prospects. Psychology of Sport and Exercise, 10(4), 420-426. https://doi.org/10.1016/j.tsc.2012.03.005

Moreno, A., Moreno, M. P., García-González, L., Gil, A., \& Del Villar, F. (2010). Desarrollo y validación de un cuestionario para la evaluación del conocimiento declarativo en voleibol. Motricidad. European Journal of Human Movement, 25, 183-195.

Moya, J. M., Villagra, H. A., \& García, M. (6 de Mayo de 2006). Valoración de la técnica, táctica y condición física de los jugadores de fútbol sala con discapacidad intelectual. Recuperado el 18 de 02 de 2016, de http://altorendimiento.com/valoracion-de-la-tecnica-tactica-y-condicionfisica-de-los-jugadores-de-fútbol-sala-con-discapacidad-intelectual/

Nunnally, J. C., \& Bernstein, I. H. (1978). Psychometric theory. New York: McGraw-Hill.

Oliver, M. (1992). Changing the Social Relations of Research Production? Disability, Handicap \& Society, 7(2), 101-114. https://doi.org/10.1080/02674649266780141

Pérez-Tejero, J., Pinilla, J., \& Vanlandewijck, Y. (2015). Perfil de rendimiento en el campeonato del mundo de baloncesto (ankara 2013) para personas con discapacidad intelectual: implicaciones en el sistema de elegibilidad. Revista Iberoamericana de psicología del ejercicio y el deporte, 10(2), 187-192.

Pérez-Tejero, J., Polo-Más, I., Pinilla, J., \& Coterón-López, J. (2017). Coaches' and referees' opinion about the influence of intellectual impairment on fundamental basketball activities. Psychology, Society, \& Education, 9(3), 469-480. DOI: 10.25115/psye.v9i3.1027.

Pinilla, J., Pérez-Tejero, J., \& Van Biesen, D. (2017). Basketball game related statistics that discriminate between players with intellectual impairment and able-bodied players. Revista de psicología del deporte, 26(3), 113119.

Pinilla, J., Pérez-Tejero, J., Sampedro, J., Refoyo, I., Lorenzo, A., Lorenzo, J., . . . Vanlandewijck, Y. (2016). Influence of intellectual impairment (II) on 
basketball players capacity to solve a game situation: towards evidencebased classification systems in II-basketball. Psychology, Society \& Education, 8(2), 121-134. DOI: 10.25115/psye.v8i2.453.

Pinilla, J., Pérez-Tejero, J., Van Biesen, D., \& Vanlandewijck, Y. (2016). Effect of Intellectual Impairment on Basketball Game-Related Statistics. Collegium antropologicum, 40(4), 279-284.

Raab, M. (2003). Decision making in sports: influence of complexity on implicit and explicit learning. International Journal of Sport and Exercise Psychology, 1(4), 310-337. https://doi.org/10.1080/1612197X.2003.9671728

Raykov, T. (1997). Estimation of composite reliability for congeneric measures. Applied Psychological Measurement, 21(2), 173-184. https://doi.org/10.1177/01466216970212006

Raykov, T., \& Marcoulides, G. A. (2015). A direct latent variable modeling based method for point and interval estimation of coefficient alpha. Educational and Psychological Measurement, 75(1), 146-156. https://doi.org/10.1177/0013164414526039

Revelle, W., \& Zinbarg, R. E. (2009). Coefficients alpha, beta, omega and the glb: comments on Sijtsma. Psychometrika, 74(1), 145-154. https://doi.org/10.1007/s11336-008-9102-z

Ruiz-Pérez, L. M., \& Arruza, J. (2005). El proceso de toma de decisiones en el deporte: Clave de la eficiencia y el rendimiento óptimo. Barcelona: Paidós.

Ruiz-Pérez, L., \& Graupera, J. (2005). Dimensión subjetiva de la toma de decisiones en el deporte. desarrollo y validación del cuestionario CETD de estilo de decisión en el deporte. European Journal of Human Movement(14), 95-107.

Skjong, R., \& Wentworth, B. H. (2001). Expert judgment and risk perception. The Eleventh International Offshore and Polar Engineering Conference. IV, págs. 537-544. Stavanger, Norway: International Society of Offshore and Polar Engineers.

Van Biesen, D., Mactavish, J., \& Vanlandewijck, Y. (2014). Tactical proficiency among table tennis players with and without intellectual disabilities. European Journal of Sport Science, 14(5), 403-409. DOI: 10.1080/17461391.2013.825645

Van Biesen, D., Mactavish, J., Kerremans, J., \& Vanlandewijck, Y. (2016). Cognitive Predictors of Performance in Well-Trained Table Tennis Players With Intellectual Disability. Adapted Physical Activity Quarterly, 33(4), 324337. DOI: 10.1123/APAQ.2015-0122.

Viciana, J., Mayorga-Vega, D., Guijarro-Romero, S., \& Martínez-Baena, A. (2017). Effect of two alternated teaching units of invasion team sports on the tactical learning in primary schoolchildren. Journal of Performance Analysis in Sport, 17(3), 256-270. https://doi.org/10.1080/24748668.2017.1331575

Viladrich, C., Angulo-Brunet, A., \& Doval, E. (2017). Un viaje alrededor de alfa y omega para estimar la fiabilidad de consistencia interna. Anales de Psicología, 33(3),

755-782. http://dx.doi.org/10.6018/analesps.33.3.268401

Voutilainen, P., \& Liukkonen, A. (1995). Senior Monitor - laadun arviointimittarin sisällön validiteetin määrittäminen. Hoitotiede, 7(1), 51-56. 
Wehmeyer, M. L., \& Obremski, S. (2010). La deficiencia intelectual. Obtenido de International Encyclopedia of Rehabilitation.: http://cirrie.buffalo.edu/encyclopedia/article.php?id=15\&language $=e s$

World Medical Association. (2013). World Medical Association Declaration of Helsinki Ethical Principles for Medical Research Involving Human Subjects. Journal of the American Medical Association, 310(20), 21912194.

Zhang, Z., \& Yuan, K. H. (2016). Robust coefficients alpha and omega and confidence intervals with outlying observations and missing data: Methods and software. Educational and Psychological Measurement, 76(3), 387411. https://doi.org/10.1177/0013164415594658

Zinbarg, R. E., Revelle, W., Yovel, I., \& Li, W. (2005). Cronbach's a, Revelle's $\beta$, and McDonald's $\omega \mathrm{H}$ : Their relations with each other and two alternative conceptualizations of reliability. Psychometrika, 70(1), 123-133. https://doi.org/10.1007/s11336-003-0974-7

Número de citas totales / Total references: 74 (100\%)

Número de citas propias de la revista / Journal's own references: $0(0 \%)$

Rev.int.med.cienc.act.fís.deporte - vol. 20 - número 79 - ISSN: 1577-0354 